Pecvnia, 1 (2005), pp. 67-92

\title{
Importancia de los planes de reordenación en la reconversión del carbón en España
}

\author{
Nuria González Rabanal
}

\begin{abstract}
El objetivo de este artículo es conocer el funcionamiento del sector minero energético a través de los sucesivos planes de reordenación del sector desde una perspectiva normativa e institucional. Estos planes de reordenación y reajuste nacen, en un principio, de la planificación energética nacional. La adhesión de España al proyecto comunitario introdujo nuevas directrices en actividades como la de la extracción de carbón. Los objetivos fijados en cada unos de ellos y articulación de los mecanismos de ayuda por ellos establecida, nos ayudarán a entender el papel desempeñado por las instituciones políticas, sociales y económicas involucradas en dicho proceso.
\end{abstract}

Palabras clave: Reconversión del carbón, sector energético, planificación energética.
The aim of this paper is the analysis of the Spanish coal rationalization plans from a normative and institutional view. The energy demand has been influenced by the economic situation of coal sector also the control of electricity companies. In the beginning, these action plans were a part of the national energy strategy. After Spanish integration in European Community, these plans were integrated into the European energy strategy. The study of each plan and their implementation help us to understand the complexity of coal rationalization process in Spain and also the role of partners' involved in the process.

Key words: Coal rationalization, energy sector, energy planning.

El Tratado Constitutivo CECA y las sucesivas Decisiones sobre intervenciones financieras de los Estados miembros en favor del carbón, así como los planes RECHAR han vertebrado, de manera directa o 
indirecta, las líneas de reconversión de la minería energética comunitaria. Una aproximación a sus contenidos revela la ausencia casi total de una estrategia clara hasta el momento en el que España se integra en la Comunidad Europea, todo ello, a pesar de la existencia de antecedentes que evidencian una cierta voluntad de apoyar la mejora en la competitividad de los carbones ${ }^{1}$. La adhesión al proyecto comunitario forzó un ajuste normativo que ha culminado en la aprobación y aplicación de sucesivos regímenes para el carbón cuyo contenido entendemos es determinante para entender los rasgos diferenciadores y el desarrollo del proceso de reconversión minero-energético español.

\section{CONVENIDA}

\section{EL RÉGIMEN DE ACCIÓN CONCERTADA y DE ACCIÓN}

Con el fin de favorecer la producción nacional de carbón, y en el marco del Plan Energético Nacional se aprueba, en 1974, un sistema de medidas de apoyo al carbón conocido como Régimen de Acción Concertada regulado por el R.D. de nueve de agosto. En este Real Decreto, se establecían unos objetivos para la producción de carbón a alcanzarse hasta 1979, y que deberían mantenerse o superarse en el periodo 80-95. Para cumplir con esta meta, se estableció un régimen de concierto entre las empresas de la hulla, la antracita y el lignito pardo, de tal forma, que aquellas empresas que aceptasen ese régimen de concierto quedaban obligadas a cumplir los aumentos de producción pactados.

Cada propuesta presentada ante el Ministerio debía suponer, al menos, un aumento en la producción anual equivalente a 50.000 toneladas tipo, que se valorarían de acuerdo a una fórmula que, entre otros elementos, tendría en cuenta el porcentaje de humedad y cenizas ${ }^{2}$.

\footnotetext{
1 La Comisión de Estudio de la Riqueza Hullera Nacional, en 1915, propuso, en 1915 , una serie de medidas necesarias para el inmediato desarrollo de la industria hullera española consistentes en el mantenimiento de ciertas condicione tributarias, la fijación de un régimen de bonificaciones para el transporte de carbón y la estimulación de la producción mediante la financiación de las inversiones productivas o el establecimiento de bonificaciones alas empresas productoras por aumentos en su producción. Más sobre este tema pude verse en: COMISION DE ESTUDIO DE LA RIQUEZA HULLERA NACIONAL. "Medidas necesarias para el inmediato desarrollo de la industria hullera española", Revista Minera, 1915, pp. 325-333.

2 La razón de tener en cuenta estos dos elementos responde a la necesidad de evitar el fraude. De esta manera, la humedad permite controlar los pesos de los carbones y las cenizas para conocer la calidad de combustión de los mismos.
} 
Para garantizar el cumplimiento de los compromisos adquiridos, se exigía a las empresas acogidas al sistema que acompañasen los contratos de un plan de estudio de la mina y un proyecto de reestructuración y mecanización que hiciese referencia al arranque, transporte interior y otras medidas dirigidas a asegurar a largo plazo el aumento del rendimiento del personal en el interior ${ }^{3}$.

Lógicamente, era necesaria una contrapartida para las empresas que se acogiesen a este sistema. Si las empresas corregían sus aumentos de producción, podrían acogerse a algunos beneficios que, discrecionalmente, proponía la Administración y que se podían traducir en beneficios fiscales y facilidad de acceso, bajo ciertas condiciones a préstamos financieros ${ }^{4}$.

La aplicación del régimen de acción concertada, en términos generales ${ }^{5}$, se tradujo en un aumento de la producción total de carbón en $2.275 \mathrm{Kt}$, siendo mayor el incremento para el lignito pardo. Sin embargo, en lo que se refiere a la reestructuración del sector, parece ser que, al igual que ocurrirá posteriormente con el régimen de acción convenida, los efectos sobre la estructura de la minería energética y las empresas que operaban en el sector no fueron los esperados, convirtiéndose el sistema en un nuevo mecanismo de financiación, basado más en la innovación de unos criterios de ayuda y protección supeditados al establecimiento de unas cuotas de producción y a la voluntariedad de las empresas de someterse al mismo, que en el establecimiento de unos objetivos de competitividad.

El fracaso del régimen de acción concertada dio paso al que se conoce como Régimen de Acción Convenida regulado por el R.D de

3 Excepcionalmente, la Administración podía tener en cuenta propuestas que, sin tener los aumentos de producción antes indicados, procurasen un aumento importante de la productividad. También existía la posibilidad de que presentasen varias propuestas conjuntamente varias empresas.

En concreto nos referimos a exenciones de licencia fiscal durante el periodo de instalación, posibilidad e acogerse a ciertos beneficios de apoyo fiscal a la inversión, libertad de amortización de las inversiones a realizar acordadas en el acta del concierto y sus ampliaciones posibles, así como la imposición de, reducción de hasta un 95\% de los impuestos generales sobre trasmisiones patrimoniales y actos servidumbres de paso para vías de acceso, líneas de transporte y distribución de la energía jurídicos -documentados.

5 Sólo hemos podido medir el grado de eficacia a través de las cifras de producción, ya que no nos ha sido posible conseguir información alguna acerca de qué empresas se acogieron a dicho régimen y cuáles fueron las variaciones y cambios experimentados en sus estructuras productivas. 
dieciséis de enero de 1981 coincidiendo con la revisión del PEN 78 y el cambio de objetivos a que condujo el segundo shock del petróleo. Para poder alcanzar las metas propuestas en el nuevo Plan Energético Nacional, se implantó un nuevo régimen de convenios entre la Administración y las empresas mineras. El espíritu de los convenios era el mismo que el del régimen de acción concertada debiéndose comprometer las empresas acogidas a tal acuerdo, a un incremento mínimo de la producción subterránea equivalente a 25.000 toneladas tipo, con un aumento relativo equivalente a como mínimo el $3 \%$ anual acumulativo de la producción subterránea de 1979. Una vez más, el aumento de la producción era el objetivo principal de las acciones en materia del carbón, quedando relegado a un segundo plano una reconversión profunda del mismo ${ }^{6}$. Para las explotaciones a cielo abierto, las perspectivas de producción se supeditarían a la realidad y las necesidades del mercado.

Las previsiones de aumento de la producción subterránea se realizaría en dos etapas: una con carácter vinculante para el periodo 1981-1985, y otra, entre 1986-1990, en la que sólo sería necesario mantener el mismo nivel de producción o, si era posible, aumentarlo.

La reconversión que tenía un carácter subsidiario, se convertía no en un fin sino en un medio para acceder a los beneficios propuestos por la Administración. Para que las empresas pudieran acogerse a los convenios, era necesario que presentasen un proyecto vinculante a la toma de decisión por parte de la Administración que se tradujese en mejoras tanto de la productividad de las explotaciones como de las condiciones socioeconómicas, de formación de personal en las minas, mejora del medioambiente y saneamiento de sus cuentas financieras y de resultados. A cambio de esto las empresas acogidas, cuyos proyectos estuviesen aceptados, podían acceder a medidas como el acceso al crédito oficial por un importe de hasta el $70 \%$ de las inversiones ${ }^{7}$ a realizar, subvenciones del capital para el fomento de la minería de hasta un $20 \%$ de

6 Este carácter secundario de la reconversión frente al aumento de la producción, se pone claramente de manifiesto en el propio Real Decreto en cuyo artículo primero se dice: ...no obstante, cuando circunstancias particulares del yacimiento de la zona lo aconsejen, la Administración podrá tener en cuenta propuestas individuales de empresas que, sin conseguir los aumentos mínimos de producción antes indicados, indiquen realizaciones importantes para la reestructuración de las minas o de su adecuado mantenimiento...

7 En los convenios entre las empresas acogidas al régimen de acción convenida y el Ministerio deberían figurar el plazo de ejecución de cada una de las instalaciones, obras o puesta en marcha de nuevos sistemas de exportación. El tipo de interés aplicado simple sería del $11 \%$. 
la inversión realizada, compensaciones para carbones procedentes de las empresas acogidas o ayudas para la investigación geológica o minera relativa al carbón.

Tanto en el régimen de acción concertada como en el de la acción convenida, la Administración actuó como intermediario entre las empresas mineras y las empresas eléctricas (principales demandantes). Para las primeras, la Administración fijaba la cuota de producción y la cuantía de las ayudas y, para las segundas, establecía el precio y las entregas a las centrales térmicas mediante los cupos.

\section{TÉRMICO}

\section{EL NUEVO SISTEMA DE CONTRATACIÓN DEL CARBÓN}

En 1987 nace el Nuevo Sistema de Contratación del Carbón Térmico -NSCCT. Este nuevo sistema supuso un nuevo giro hacia lo que podríamos llamar una "legalización de la discriminación entre la minería pública y privada" dentro de nuestro país. La puesta en marcha de este sistema, aún hoy vigente en su diferenciación entre empresas públicas y privadas, sirvió igualmente como base para la aplicación y el cumplimiento de la normativa comunitaria de la que ya formábamos parte.

El NSCCT fue suscrito por los representantes nacionales del carbón asociados en CARBUNION y los empresarios eléctricos a través de UNESA, con el visto bueno de la Administración. A partir de ese momento, podemos decir que se "oficializa" la ayuda indiscriminada a las empresas públicas mineras.

La menor competitividad de la industria española ya había forzado en la década de los sesenta a la incorporación al INI de algunas empresas industriales estratégicas pero que no suficientemente competitivas. En 1967 se creó HUNOSA, una empresa pública asturiana que, lejos de cumplir con los objetivos establecidos por el Estado para las empresas integradas en el INI, de sanearse para después privatizarse, ha seguido inmersa en una situación en la que las subvenciones por tonelada se han mantenido en unos niveles... "extraordinariamente altos, que representan una extraordinaria carga para el sector público..." ${ }^{8}$. Su no 
saneamiento económico ha obligado ${ }^{9}$ a la Administración a costear permanentemente sus pérdidas ${ }^{10}$ por lo que, cuando España entra en la Comunidad Europea y la necesidad de ajuste en este sector es imperativa, se decide poner en marcha un sistema que permita a las empresas suscribir dos tipos de contratos dependiendo, en primer lugar, del carácter público o privado de la explotación minera, y en segundo lugar, del volumen y capacidad de producción.

De acuerdo con el primer criterio nos encontramos con dos tipos de empresas: las empresas con contrato de programa -ECCP- que fueron las explotaciones mineras asturianas HUNOSA, Minas de FIGAREDO S.A. y la Mina LA CAMOCHA; y las empresas sin contrato de programa -ESCPconformado por el resto de las empresas mineras acogidas al sistema tanto de explotación subterránea como a cielo abierto. Para las ECCP las ayudas servirían para compensar las pérdidas y la cuantía de las mismas se haría con cargo a los Presupuestos Generales del Estado y las aportaciones del INI. En las ESCP el régimen de ayudas estaba supeditado a un régimen específico y diferenciado según el carácter de la explotación y su volumen de producción, aunque basado en un sistema de contratos a largo plazo entre las empresas productoras y las eléctricas.

Los contratos a largo plazo que podían suscribir las ESCP podían ser de dos tipos; acogidos a un precio de referencia o de precio libre a determinar entre las partes. De los contratos a largo plazo con precio de referencia podían beneficiarse las empresas mineras ${ }^{11}$ con una producción subterránea anual superior a $50.000^{12}$ toneladas-valor referidas a los poderes caloríficos de su producción en 1985. Lógicamente, a la otra modalidad -precio a determinar libremente- podrían acogerse las empresas

9 Decimos obligado porque a nuestro entender la decisión de continuar la actividad por parte de esta empresa obedece más a factores puramente políticos que socioeconómicos, ya que HUNOSA se ha beneficiado de subvenciones y ayudas muy superiores a las del resto de las empresas mineras desde hace más tiempo que éstas, y con peores resultados en términos de competitividad.

10 Tal y como señalan J.A. Vázquez, y S.R. Martinez (Ob. Cit ), la continuidad de HUNOSA ha dependido tanto de la protección practicada a los carbones térmicos y siderúrgicos a través de la fijación administrativa de los precios y las restricciones a la importación, como de la concesión de importantes ayudas estatales.

11 Podían acogerse a este sistema también asociaciones de empresas, siempre que dicha asociación se realizase en condiciones de responsabilidad solidaria y conjunta y se ajustasen al resto de requisitos impuestos por el sistema de contratación.

12 A estas empresas se les garantizaba, para 1987, unas entregas térmicas semejantes a las efectuadas en 1986. Las entregas irían disminuyendo progresivamente pasando el resto de la producción termoeléctrica al mercado libre. 
con una cuota de producción inferior a las 50.000 toneladas valor que realizasen su extracción bien por laboreo o a cielo abierto. Las condiciones exigidas por el sistema eran claramente diferentes en uno y en otro caso. A las empresas mineras que se acogiesen al sistema de precios de referencia, se les exigía la presentación, previa aprobación del contrato, de un plan estratégico que demostrase no sólo la viabilidad técnica, sino también la económica de las explotaciones a largo plazo, junto con una auditoria contable del último ejercicio. En el plan estratégico debía figurar no sólo un análisis de las reservas explotables por la empresa, sino también un plan de explotación para los años de duración del contrato, un estudio sobre la evolución previsible de la plantilla empleada, y un plan de inversiones y financiación con las cuentas financieras anuales de la empresa. Todos estos elementos tenían como finalidad el permitir a la Administración dotarse de ciertas garantías que asegurasen la consecución de la eficiencia empresarial y el ajuste y reducción de capacidad ${ }^{13}$ que eran necesarios.

El diseño de un sistema como el descrito debía ofrecer, al igual que ocurrió con otras fórmulas anteriores, una contraprestación tanto a las empresas mineras como a las eléctricas. A todas las empresas acogidas al sistema de precios de referencia cuya cuenta de pérdidas y ganancias resultase negativa ${ }^{14}$, se les reconocía el derecho a solicitar de las compañías eléctricas, con las que hubieran suscrito el contrato a largo plazo, un suplemento de precio sobre el precio de referencia ${ }^{15}$. A su vez, las compañías eléctricas solicitarían de la Administración la aprobación de dicho complemento para que OFICO (Oficina para la Compensación de la Energía Eléctrica) compensase a las compañías eléctricas ${ }^{16}$ las cantidades que éstas habían abonado a las mineras en concepto de sobreprecio.

13 Trianualmente se revisarían las cantidades acogidas al precio de referencia al actualizar el plan estratégico de cada empresa. Anualmente, las empresas eléctricas y las mineras, independientemente, presentarían un informe anual a la Administración sobre el desarrollo y las incidencias derivadas de la ejecución del contrato que, una vez negociado entre las partes, se presentaba ante la Administración. participaban de este sistema.

14 Exceptuando, lógicamente, las que tenían contrato de programa, que no

15 Para poder acceder a dicho suplemento de precio, las empresas mineras debían presentar ante la empresa eléctrica una auditoria técnica y económica del último ejercicio contable. Como podía darse el caso de que una misma empresa tuviese explotación subterránea y a cielo abierto, en ese caso, se admitía presentar dicha auditoria de forma integrada para ambos tipos de explotación.

16 OFICO liquidaría las compensaciones a las empresas que estuvieran a cogidas al SIFE (Sistema Integrado de Facturación de la Energía Eléctrica). 
La compensación para las compañías eléctricas no era única, estableciéndose que podían obtenerlas por los siguientes conceptos:

a. - Compensaciones para todos los carbones a centrales térmicas por la diferencia entre el valor estándar y el límite del coste.

b. - Compensaciones de coste por adquisición u obtención de carbones procedentes de las explotaciones subterráneas que excediesen a los derivados de los precios acordados en los contratos a largo plazo o planes de suministro.

c.- Compensaciones por almacenamiento ${ }^{17}$, en cuyo caso la Delegación de Gobierno fijaría, para cada central, las producciones eléctricas mínimas necesarias para garantizar conjuntamente el consumo de los carbones con derecho a compensación por almacenamiento, de las existencias de carbón adquiridas con anterioridad al 1 de enero de 1987, así como los procedentes de empresas acogidas al sistema de referencia de precios u otro suministro que excediese las necesarias para un consumo de 720 horas de utilización a plena carga.

d.- Compensaciones de gastos de reducción de emisiones contaminantes fijadas por el Ministerio de Industria en el marco de las iniciativas comunitarias.

e.- Compensación por transporte en aquellos casos en los que se estimase que los carbones debían ser transportados a las centrales alejadas de las cuencas mineras de procedencia.

Todo este sistema de compensaciones a las eléctricas, no impedía la importación de carbones de otros países, simplemente garantizaba el consumo de carbón nacional y establecía algunas condiciones para la importación ${ }^{18}$.

En el momento de ponerse en marcha el sistema se estableció que, para ese año, el precio base de referencia fuese el correspondiente

17 Dicha compensación se calcularía mensualmente como un \% del valor medio de las existencias que a tal tuvieran derecho al finalizar el mes anterior.

18 En el caso de la importación de hulla, las empresas eléctricas ponían a disposición de OFICO la diferencia entre un límite del coste estándar expresado en pesetas termia referidas al poder calorífico superior y un precio estándar determinado para cada partida expresado también en pesetas termia y calculado para un mes con la media ponderada de los precios CIF expresados en \$ USA/tonelada de hulla. Además, las empresas eléctricas no podían utilizar combustibles sólidos importados con un contenido de azufre superior al $0,10 \%$ por cada mil termias/tonelada de poder calorífico superior sobre muestra bruta. 
al valor estándar unitario fijado para el año 1986, incorporándose un suplemento de precio del $2,5 \%$ que sería actualizado de acuerdo con una fórmula de revisión que se aplicaría cada año.

Para las empresas que no podían acogerse al sistema de precios de referencia, los precios se determinarían libremente entre los consumidores y productores dentro de unas pautas marcadas por la Administración. Con carácter general, las empresas eléctricas darían preferencia al consumo de carbón nacional siempre que dispusiesen de una oferta adecuada con dos excepciones: una, las centrales térmicas del sur de España para las que se admitía un límite del 80\% de importaciones de sus necesidades actuales, y otra, para las centrales con problemas medioambientales, que podrían consumir carbón de importación o gas en función de las condiciones específicas de contaminación.

El nuevo sistema implantado tuvo consecuencias sobre el número de explotaciones en funcionamiento. Desde 1986 hasta 1990 esta cifra ha pasado de 225 a 163. El pago de suplementos de precio para empresas acogidas a precios de referencia superó al de las empresas no acogidas al mismo, que vieron reducidas sus cuotas de producción.

RESUMEN RESULTADOS DEL PLAN 86-90

\begin{tabular}{|l|c|c|c|}
\hline & $\begin{array}{c}\text { PAGOS } \\
\text { (M. Ptas.) }\end{array}$ & $\begin{array}{c}\text { BAJAS } \\
\text { LABORALES }\end{array}$ & $\begin{array}{c}\text { PRODUCCIÓN } \\
\text { (KT) }\end{array}$ \\
\hline SUPLEMENTOS DE PRECIO & 33.627 & 0 & 0 \\
\hline CIERRES Y AJUSTES DE PLANTILLA & 19.865 & 1.285 & 472 \\
\hline
\end{tabular}

Fuente: OFICO. El carbón nacional: situación actual e hipótesis de futuro. Reflexiones sobre un nuevo marco en el sector. Ministerio de Industria. 1996, y elaboración propia.

La aplicación del NSCCT no respondió a las expectativas de reestructuración y racionalización pretendidas al comienzo de su aplicación. En 1998, el coste de la tonelada vendible de carbón leonés era de 61,42 euros frente a los 120,80 euros por tonelada de HUNOSA o los 98,66 euros por tonelada de Alemania, lo que pone de manifiesto que, la minería de León, en esos momentos era de las más competitivas a pesar de una política estatal discriminatoria que permitió una financiación, en concepto de ayudas para la minería pública, de 60.000 millones de pesetas anuales frente a los 5.000 millones anuales percibidos por la leonesa.

A la vista de lo anterior y teniendo en cuenta las valoraciones hechas tanto desde las empresas mineras como de la Administración 
regional, el NSCCT no cumplió con los objetivos propuestos acentuando las divergencias entre las explotaciones mineras. El sistema formulado respondió más, a nuestro entender, a la necesidad de cumplir con la adaptación a la normativa comunitaria que a las necesidades reales del sector. De repente era necesario sanear las empresas mineras en pos de conseguir su adaptación a un mercado más abierto, y alcanzar la consecución de cuotas de producción no sólo afianzadas en yacimientos significativos, sino también extraíbles a costes razonables. Dadas las condiciones socioeconómicas de las cuencas mineras, garantizar de forma estable el mayor empleo posible era un claro objetivo político. Que todo ello fuese coherente con un aumento gradual de la viabilidad económica y un ajuste racional a la demanda y oferta previsibles, nos parece una meta tremendamente difícil de conseguir, por no decir que imposible. Lo cierto es que el NSCCT parecía dar solución a la garantía de la demanda con el sistema de contratos a largo plazo. De esta manera se aseguraba para las empresas mineras unas compras que les proporcionaban no sólo estabilidad en la producción, sino también la posibilidad de ejecutar inversiones en sus explotaciones y beneficiarse de las facilidades que, a tal fin, la Administración estaba dispuesta a conceder siempre que se cumpliesen determinadas condiciones.

El consumo de carbón nacional distribuido principalmente entre la siderurgia, las coquerías y las centrales térmicas, posicionaba a las empresas eléctricas en una situación de privilegio frente al resto de consumidores, lo que les permitió beneficiarse por encima de los que no contaban con posibilidad de financiación complementaria alguna por sus compras de carbón nacional.

ESTRUCTURA DEL CONSUMO NACIONAL DE CARBÓN EN 1987 (Kt)

\begin{tabular}{|l|c|c|}
\hline SECTORES & $\begin{array}{c}\text { HULLA Y } \\
\text { ANTRACITA }\end{array}$ & $\begin{array}{c}\text { LIGNITO } \\
\text { NEGRO }\end{array}$ \\
\hline SIDERURGIA Y COQUERÍAS & 3.799 & \\
\hline CENTRALES TÉRMICAS & 16.897 & 5.653 \\
\hline CEMENTO & 2.260 & 2 \\
\hline RESTO INDUSTRIAS & 1.450 & 90 \\
\hline
\end{tabular}

Fuente: Elaboración propia a partir de la información facilitada por OFICO. 


\section{EL PLAN DE REORDENACIÓN DEL CARBÓN 1990-1993}

En 1990, y con motivo del requerimiento de la Comisión Europea a España ${ }^{19}$ a presentar una plan de reducción de los pagos compensatorios efectuados en el marco del NSCCT aplicable hasta 1993, se diseña un Plan de reordenación para el sector del carbón que se aplicaría, en un principio, para el periodo 1990-1993, y que posteriormente se vería prorrogado hasta 1994. Aunque se seguiría manteniendo el sistema de contratos a largo plazo, se establecieron nuevos objetivos y medidas de apoyo. Dicho plan se estructuró en torno a cinco ejes: reducir los costes de producción del carbón nacional mediante una disminución progresiva de las ayudas del Estado, alcanzar una mejora de la productividad media en la minería subterránea de un $15 \%$, consolidar una capacidad de la minería a cielo abierto de unas $600 \mathrm{Kt}$ al final del plan, mejorar los niveles de seguridad minera y promover la regeneración del tejido industrial en las áreas afectadas por los cierres de capacidades mineras.

La previsiones en cifras de los objetivos establecidos para la minería subterránea, reflejaron reducciones muy importantes en el número de trabajadores empleados en el sector $(-28 \%)$, de empresas $(-37 \%)$ y de los suministros globales a las empresas termoeléctricas, lo que hizo ascender el coste estimado total de la reordenación prevista a 125.000 millones de pesetas del año 90 .

PREVISIONES PLAN DE REORDENACIÓN 90-93

\begin{tabular}{|l|c|c|}
\hline & $\mathbf{1 9 8 9}$ & $\mathbf{1 9 9 4}$ \\
\hline PLANTILLA & 22.773 & 16.604 \\
\hline SUMINISTRO TÉRMICO & 10.811 & 9.387 \\
\hline $\mathrm{N}^{0}$ EMPRESAS & 172 & 107 \\
\hline PRODUCTIVIDAD (T/H/A , O) & 475 & 572 \\
\hline COSTE MEDIO (Ptas. 89/Th PCS) & 2,274 & 2,051 \\
\hline
\end{tabular}

Fuente: Elaboración propia a partir de los datos ofrecidos por el Ministerio de Industria.

El instrumento a través del cual se pone en marcha es la Real Orden Ministerial de 31 de octubre de $1990^{20}$ en la que se plasman

19 BOE, miércoles 7 de noviembre de 1990.

20 Decisión 20.12.89 DOCE L /105/19. En el V considerando de dicha Decisión se señala que, para que la Comisión estuviese en condiciones de poder aplicar la Decisión 2064/86 CECA, 
las principales medidas de reducción de los pagos compensatorios que conducían paralelamente a una reducción de capacidades.

Las empresas explotadoras de las centrales térmicas y las mineras podrían acceder al pago de compensaciones como consecuencia de contratos visados por la Secretaría de Energía, siempre que los pagos tuviesen el carácter compensatorio por una reducción no inferior al $40 \%$, según promedio anual, de los niveles de suministro hasta 1993. La posibilidad de beneficiarse de las percepciones por este concepto, lo hacía incompatible con los pagos de suplementos de precio aunque existía la posibilidad de acceder, bajo ciertas condiciones ${ }^{21}$ a un anticipo del suplemento de precio para aquellas empresas suministradoras de carbón térmico que estuvieran realizando un cambio sustancial en la aplicación de los factores de producción que les permitiese obtener un saldo positivo en la cuenta de explotación. La compensación por reducción de la producción tenía, a su vez, dos componentes; uno laboral ${ }^{22}$ y otro de reducción de la producción, condicionado por el primero.

\section{PLAN DE REDUCCIÓN DE ACTIVIDAD DE LA INDUSTRIA DEL} CARBÓN ESPAÑOLA 1994-1997

El final de la vigencia de la Decisión 2064/86 CECA y la puesta en marcha de la Decisión 3632/93 CECA obligan a un nuevo ajuste de nuestro sistema de subvención a la minería. La reclasificación del tipo de ayuda, la homologación en el tratamiento de las explotaciones a cielo abierto y los graves problemas socioeconómicos de las cuencas mineras en declive obligaron a las autoridades españolas a reformar, una vez más, el sistema de contratos a largo plazo introduciendo algunas novedades. El nuevo sistema se reflejó en un nuevo Plan de modernización, racionalización y reestructuración y reducción de actividad de la industria del carbón española 1994-1997.

\footnotetext{
se sugiere a las autoridades españolas la presentación, antes del treinta de junio, de un plan de reducción de los pagos compensatorios efectuados en el marco del NSCCT o de cualquier otra intervención de efecto equivalente.

21 La Orden Ministerial establecía que para poder acceder al anticipo de precio era necesario demostrar la viabilidad de las empresas en el plan estratégico que debían adjuntar las empresas suministradoras a las empresas generadoras de electricidad visado por la Secretaría de Energía.

22 La componente laboral respondía a un acuerdo alcanzado entre las empresas y los sindicatos con el visto bueno de la Administración.
} 
El nuevo plan insistía en la reducción de las ayudas a las empresas productoras de carbón por la venta del mismo. Lógicamente, y una vez más, se mantenía la diferencia entre las empresas con contrato de programa y las empresas sin contrato, en lo que creemos una obstinada intención de diferenciar entre la minería pública y la minería privada, aunque en la declaración de intenciones del propio plan parecía aflorar una cierta voluntad de reducir las ayudas también para las públicas. Tres eran los ejes sobre los que se vertebraba este nuevo sistema regulador del carbón:

El primero era la continuidad de las ayudas al funcionamiento, condicionadas, eso sí, a la consecución de una mejora de la productividad. Si con anterioridad los ingresos que percibían las empresas mineras llevaban intrínseca una ayuda consistente en percibir una cantidad sobre el coste del carbón internacional puesto en central, a partir de ese momento, ese ingreso crecería dos puntos porcentuales por debajo del crecimiento del índice real de precios al consumo, lo que garantizaba una reducción de las ayudas en un 6,31\% como mínimo. Las explotaciones a cielo abierto, también verían reducidos sus ingresos, y las cantidades a suministrar se pactarían anualmente en función de las necesidades de las centrales térmicas.

El segundo, era el constituido por las ayudas a la reducción de actividad, que se concederían inevitablemente a las empresas con contrato de programa que, a todas luces, era imposible que tuvieran unos costes de producción que se aproximasen a los del mercado internacional ${ }^{23}$, y a las empresas sin contrato de programa para las que se determinarían las posibilidades financieras a las que tendrían acceso aquellas que redujesen total o parcialmente toda la actividad.

El tercero, las ayudas a la reindustrialización mediante la creación de un fondo económico que permitiría financiar las actuaciones dirigidas a establecer alternativas económicas a la minería energética en las cuencas afectadas por la reducción de actividad.

Para poder acogerse al sistema de ayudas, las empresas debían presentar un plan de reducción de actividad que reflejase tanto

23 La severidad en el grado de reducción de actividad para las empresas con contrato de programa era la máxima que permitían las condiciones sociolaborales de las cuencas mineras con grandes dificultades para el establecimiento de inversiones alternativas a la minería. 
las reducciones de los ingresos por ventas de carbón, como las de las plantillas y los previsibles cierres de explotaciones y aumentos de productividad previstos. El plan distinguía entre las ECCP y las ESCP, y dentro de este segundo grupo, entre las empresas con un volumen de producción superior a 200.00 toneladas o inferior a este valor, así como entre explotaciones subterráneas y a cielo abierto.

Para las empresas con contrato de programa se estipuló que la lejanía irreductible entre sus costes y los del mercado internacional, justificaban la prolongación de las ayudas concedidas más allá del horizonte temporal marcado por el fin de la vigencia del Tratado CECA y la Decisión 3632/93 CECA, aunque se cumpliría progresivamente el objetivo de reducción de las mismas.

Las empresas sin contrato de programa eran 95, de las cuales 24 simultaneaban la explotación a cielo abierto con la explotación subterránea. Diez de estas empresas tenían capacidad de entrega superior a las 200.000 toneladas anuales llegando su producción anual a las 5.410.250 Tm lo que daba empleo a 7.343 trabajadores; las ochenta y cinco restantes entregaban una producción total de $4.441 .000 \mathrm{Tm}$ y empleaban a casi tantos trabajadores como las otras diez (7.046).

Un panorama como el descrito impedía cumplir con la idea de crear un plan para cada una de las empresas, por lo que la Administración optó por utilizar un criterio de homogeneización por cuencas mineras.

Para las empresas sin contrato programa, la meta era la reducción de actividad con disminución de los costes de producción y los ingresos por ventas de carbón a las centrales térmicas. El criterio de reducción del ingreso sería el mismo que para las ECCP, es decir, una disminución de dos enteros porcentuales a la elevación experimentada por el IPC ${ }^{24}$.

Las empresas con producción por encima de las 200.000 Tm presentaron un plan de reducción de costes empresa por empresa.

24 De este modo, para mantener su rentabilidad, las empresas debían reducir como mínimo sus costes un $8,62 \%$ durante la vigencia del plan, lo que implica una tasa anual de inflación del 3,5\% para todo el plan. 
RESUMEN DE PLANES DE REDUCCIÓN DE COSTES DE EMPRESAS DE MÁS DE 200.000 TONELADAS ANUALES

\begin{tabular}{|c|c|c|c|c|c|c|c|c|c|}
\hline EMPRESAS & $\begin{array}{l}\text { SUMINISTRO } \\
\text { CONTRATADO }\end{array}$ & $\begin{array}{c}\text { PLANTILLA } \\
94\end{array}$ & $\begin{array}{c}\text { PLANTILLA } \\
97\end{array}$ & $\begin{array}{c}\text { DIFERENCIAL } \\
94-97\end{array}$ & \begin{tabular}{|c} 
REDUCCIÓN \\
DE COSTES \\
$94-97$ \\
Pta/Th kte. \\
\end{tabular} & INVERSIÓN & \begin{tabular}{|c|} 
AYUDA \\
UNITARIA \\
INTRÍNSECA
\end{tabular} & \begin{tabular}{|c|} 
AYUDA \\
UNITARIA \\
TOTAL \\
M Ptas \\
\end{tabular} & $\begin{array}{c}\text { SUBVENCIONES } \\
\text { A LA } \\
\text { INVERSIÓN }\end{array}$ \\
\hline M.S.P. & 1.300 .000 & 2.323 & 1.859 & 464 & $2,720-2,054$ & 8.838 & 1.090 & 1.246 & 12.072 \\
\hline VICTORIANO ALONSO & 1.022 .000 & 1.352 & 1.341 & 11 & $2,137-2,040$ & 14.297 & 1.011 & 1.033 & 1.190 \\
\hline H.V.L.S.A. & 960.000 & 1.539 & 1.641 & -102 & $2,150-2,011$ & 18.418 & 4.485 & 4.306 & 1.015 \\
\hline H. COTO CORTES & 493.500 & 611 & 583 & 28 & $1,820-1,778$ & 2.720 & 998 & 492 & 143 \\
\hline S.A.M.C.A. & 385.000 & 274 & 267 & 7 & $\frac{2,285-2,172}{2,23}$ & $\begin{array}{l}1.252 \\
1.252\end{array}$ & 3.292 & 1.267 & 0 \\
\hline ENDESA & 373.000 & 639 & 485 & 154 & $4,617-4,126$ & 783 & 2.575 & 960 & 0 \\
\hline ARAGON MINERA & 274.000 & 125 & 120 & 5 & $2,218-2,218$ & 535 & 3.517 & 964 & 0 \\
\hline PEDRAFORCA & 257.250 & 154 & 152 & 2 & $2,108-1,916$ & 764 & 3.699 & 951 & 114 \\
\hline ANTRACITAS GUILLON & 245.000 & 453 & 438 & 15 & $2,145-1,938$ & 725 & 3.966 & 972 & 145 \\
\hline ENCASUR & 200.000 & 391 & 312 & 79 & $2,967-2,533$ & 1.009 & 430 & 86 & 91 \\
\hline TOTAL & 5.509 .750 & 7.861 & 7.198 & 663 & & 49.341 & & 12.277 & 14.770 \\
\hline
\end{tabular}

Fuente: Plan de modernización, racionalización y reestructuración y de reducción de actividad de la industria del carbón española 1994-1997 y elaboración propia.

Para las empresas con cuotas de producción inferiores a las 200.00 toneladas la solución no podía ser otra que la reducción de actividad mediante la aplicación de un sistema de prejubilaciones, jubilaciones anticipadas y bajas incentivadas. El modo de hacerlo era contando con que la Administración se comprometiese a financiar las bajas y jubilaciones siempre que la empresa acogida redujese progresivamente sus entregas de carbón a las centrales térmicas. El objetivo último era disminuir los ingresos por ventas de carbón para estas empresas en un $20 \%$ en cuatro años.

El coste total del plan para las empresas sin contrato de programa ascendería a 71.818 millones de pesetas, de los cuales 49.746 serían aportaciones de OFICO para financiar tanto costes sociales como ayudas a la reestructuración de pasivos e industrialización; el resto -22.072se ejecutarían vía Presupuestos Generales del Estado. Un resumen del desglose presupuestario es el que ofrecemos en el cuadro adjunto.

COSTE DEL PLAN PARA LAS ESCP (Millones de pesetas)

\begin{tabular}{|c|c|c|c|c|c|c|c|}
\hline & & \\
\hline & & & 1994 & 1995 & 1996 & 1997 & TOTAL \\
\hline \multirow{5}{*}{$\begin{array}{l}\text { INTERVENCIÓN } \\
\text { FINANCIERA } \\
\text { OFICO }\end{array}$} & \multirow{2}{*}{$\begin{array}{l}\text { COSTES } \\
\text { SOCIALES }\end{array}$} & JUBILACIONES & 7.030 & 4.750 & 4.750 & 2.204 & 18.734 \\
\hline & & BAJAS INCENTIVADAS & 2.845 & 1.815 & 1.815 & 1.122 & 7.597 \\
\hline & \multicolumn{2}{|c|}{ AYUDA REESTRUCTURACIÓN PASIVOS } & 5.870 & 3.790 & 3.790 & 2.160 & 15.610 \\
\hline & \multicolumn{2}{|c|}{ AYUDA REINDUSTRIALIZACIÓN } & 2.935 & 1.895 & 1.895 & 1.080 & 7.805 \\
\hline & \multicolumn{2}{|l|}{ TOTAL } & 18.680 & 12.250 & 12.250 & 6.566 & 49.746 \\
\hline
\end{tabular}




\begin{tabular}{|l|l|r|r|r|r|r|}
\cline { 2 - 7 } \multicolumn{2}{c|}{} & $\mathbf{1 9 9 4}$ & $\mathbf{1 9 9 5}$ & $\mathbf{1 9 9 6}$ & $\mathbf{1 9 9 7}$ & \multicolumn{1}{c|}{ TOTAL } \\
\hline \multirow{2}{*}{$\begin{array}{l}\text { PRESUPUESTOS } \\
\text { GENERALES } \\
\text { DEL ESTADO }\end{array}$} & M.S.P. & 4.480 & 4.046 & 3.546 & 0 & 12.072 \\
\cline { 2 - 7 } & AYUDAS A INVERSIÓN & 2.500 & 2.500 & 2.500 & 2.500 & 10.000 \\
\cline { 2 - 7 } & TOTAL PGE & 6.980 & 6.546 & 6.046 & 2.500 & 22.072 \\
\hline
\end{tabular}

Fuente: Plan de modernización, racionalización, reestructuración y reducción de actividad de la industria del carbón española y elaboración propia.

El matiz diferenciador entre las explotaciones a cielo abierto y las de laboreo surge en el momento en el que se comprueba no sólo la existencia de unas capacidades muy diferenciadas entre las empresas que explotan en este régimen, sino también en las condiciones por ellas pactadas para las entregas de los carbones. Aunque a partir de enero de 1994 los precios de referencia del carbón fueron los mismos para la minería subterránea que a cielo abierto, estaba claro que este tipo de minas estaban sometidas a unos criterios más rígidos que el resto existiendo, por tanto, una ayuda implícita menor. Asumiendo este hecho, el plan prevé en el marco de la Decisión 3632/93 CECA, que los ingresos de estas empresas llevasen la misma reducción que la minería subterránea (dos enteros porcentuales por debajo del IPC). A diferencia de la minería subterránea, éstas tendrían que reducir sus ingresos, pero no los suministros debido a la mayor flexibilidad de su parada o arranque.

A pesar de los esfuerzos por un ajuste, en 1997 se realizó una valoración de la ejecución de los mismos, que serviría para poder configurar el plan del carbón 1998-2005. Como podemos observar en los cuadros adjuntos, ni las empresas con contrato de programa ni las sin contrato de programa pudieron cumplir con los objetivos propuestos a nivel presupuestario, de producción y empleo. El análisis que se realizó fue más exhaustivo para las empresas con contrato de programa que para las sin contrato de programa.

La situación no fue muy distinta para las empresas sin contrato de programa. Aunque no hemos podido contrastar los datos previstos en los planes de reducción de actividad de cada empresa, sí que podemos evaluar en su conjunto la evolución. La reducción prevista de la producción en 1993 para 1997 fue de 1.561 millones de toneladas, sin embargo en 1996 sólo se había conseguido disminuir en 427. Por lo que se refiere al empleo, la reducción prevista fue de 3.890 trabajadores y la conseguida de 769 .

A la vista de los anteriormente mostrado, parece evidente que en nuestra minería energética no se han cumplido con rigor los 
compromisos adquiridos, y que no existen diferencias sustanciales entre lo que han hecho las empresas públicas mineras y las privadas.

\section{PLAN PARA LA MINERÍA DEL CARBÓN 1998-2005}

El Plan de reordenación finalizó en 1997, coincidiendo con la aplicación de la Directiva Europea del Mercado Interior de la Electricidad ${ }^{25}$ que permite la búsqueda de compatibilidad entre la liberación del mercado eléctrico y la seguridad del abastecimiento energético, poniendo de manifiesto la prioridad de la seguridad del mismo por encima de la optimización de $\operatorname{costes}^{26}$. Aunque dicha directiva reconocía la posibilidad de que los Estados miembros pudiesen imponer a las compañías eléctricas obligaciones de servicio público e interés económico, como puede ser la seguridad de abastecimiento, también establece un techo máximo en la utilización de fuentes de energía primarias del $15 \%$ de la cantidad total de la energía primaria necesaria para producir la electricidad que se consumiese en el Estado miembro del que se tratase. Con ello, se establece la precedencia económica en el funcionamiento de las centrales térmicas, aunque no interviene ni regula un nuevo sistema de ayudas al carbón diferente o complementario al diseñado por la Decisión 3632/93 CECA.

Es precisamente la liberalización del sistema eléctrico y la necesidad de dar continuidad al cumplimiento de las directivas comunitarias sobre el carbón junto con las permanentes reivindicaciones de los trabajadores del sector de ver garantizado su futuro, lo que lleva a la Administración a pactar con los sindicatos un Plan para la minería del carbón y desarrollo alternativo de las cuencas mineras para el periodo 1998-2005. El acuerdo no contó con la aceptación de los productores de carbón ni tampoco de los eléctricos, hecho éste que, además de restar efectividad al proceso de reestructuración, fue muy criticado en su momento por las implicaciones que para los ausentes en la negociación tendría el plan.

Básicamente se seguían las mismas líneas que en los anteriores planes ya que, para las compras de carbón, se siguió pensando que el mejor instrumento eran los cupos garantizados. Para las empresas eléctricas se establecía el principio de libre contratación, con la condición

25 Publicada el 30 de enero de 1997.

26 Artículos 3.2, 8.4 y 24.1 de la Directiva. 
de que a cambio deberían garantizar los compromisos mínimos, para lo cual se suscribirían los contratos. Como contraprestación, las eléctricas recibirían ayudas a la financiación de los stocks de carbón por encima de las 720 horas de seguridad. Los compromisos se reducen progresivamente en el tiempo, llegándose a garantizar unas compras, en el año 2005, de 13.000.000 toneladas, lo que implica una reducción acumulada del $28,32 \%$.

El plan detalla los compromisos de compra para cada una de las centrales térmicas, teniendo en cuenta que dichos compromisos no implicaban que se pudieran superar los mismos, además de los tipos de ayudas a ejecutar que serán ayudas indirectas, al funcionamiento y a la reestructuración.

Si tenemos en cuenta los compromisos fijados, podemos observar una progresiva reducción de los mismos para el periodo temporal pactado en el plan. Concretamente, la reducción total será de un $28,32 \%$, repartida en reducciones anuales que se moverán en un intervalo entre el $3,50 \%$ y un $4,71 \%$. Las cuotas comprometidas están en clara correlación con la potencia de las centrales térmicas, siendo las que mayor potencia contratada tienen las que se benefician de mayores consumos garantizados.

Las ayudas indirectas al carbón cubrirían el objetivo de financiar los stocks y trasvases de carbón desde las empresas mineras hasta los grupos de explotación. Las ayudas al funcionamiento ${ }^{27}$, cubrirían la diferencia entre los costes de producción y el precio de venta libremente acordado entre las partes ${ }^{28}$, eso sí, teniendo en cuenta las condiciones reinantes en el mercado internacional y el carácter de la explotación ${ }^{29}$ (subterránea o a cielo abierto).

\footnotetext{
27 Para las empresas que no suscribieran el plan, las ayudas se reducirían en un $10 \%$ adicional al correspondiente al importe de la ayuda.

28 Esto supone la conversión a una subvención a los costes de explotación de las empresas mineras de la ayuda que, hasta hoy, se obtiene como resultado de la diferencia entre el ingreso al minero y el precio de adquisición de las centrales térmicas, multiplicado por el cupo garantizado a cada empresa minera.

29 La ayuda calculada para las explotaciones subterráneas se reducirá un $4 \%$ anual ajustándose a un procedimiento en el que se tendrán en cuenta: un ajuste por actualización de costes en la que el máximo importe actualizado será el resultado del producto de la del año anterior por el IPC previsto con un techo máximo del 2\%; un ajuste por consumo garantizado en función del consumo garantizado para el año siguiente; un ajuste por condiciones prevalecientes en el mercado internacional y, por último, un ajuste por razones laborales en las que se condiciona la percepción de la ayuda al cumplimiento de un esquema de jubilaciones y prejubilaciones, según el cual, por cada 11 bajas deben existir 4 ingresos en plantilla fija. En caso contrario, las ayudas se reducirán un $50 \%$ a partir del 1 de enero del año 2000, suprimiéndose en el 2003. El plan también fija como máximo una reducción, mediante medidas no traumáticas, de 7.000 puestos de trabajo.
} 
El plan se ha visto complementado con un paquete de medidas que tienen como finalidad contribuir a una mejora del grado de reindustrialización de las cuencas mineras afectadas por el declive.

Trascurrido el periodo de vigencia del Plan del carbón, es necesario abordar una nueva línea de actuación. A la dificultad implícita de dicho proceso, se han de incorporar en la nueva negociación los nuevos objetivos energéticos de la UE marcados por el compromiso medioambiental de la UE de reducción de uso de fuentes de energía convencionales y por el mantenimiento de un mínimo de reservas energéticas autóctonas que han de ser consideradas "estratégicas" sin que se haya explicitado por parte de las instituciones comunitarias que parámetros y condiciones son exigibles a las explotaciones mineras para ser consideradas bajo esta denominación. En todo caso, desde la UE se ha manifestado claramente que la calificación de reserva estratégica en ningún modo garantizará el mantenimiento de la actividad minero-energética a los niveles actuales.

A modo de conclusión, podemos decir que la continuidad de la minería del carbón se ha conseguido, en nuestra opinión, mediante la compatibilidad de las garantías del consumo de carbones nacionales con las ayudas públicas. La regulación normativa de las compras de carbón a las empresas mineras por parte de las empresas eléctricas ha sido la forma más común de abordar este objetivo. Incardinar dichos acuerdos en el marco impuesto por las sucesivas Decisiones sobre intervenciones financieras en favor del carbón, no ha resultado una tarea fácil. Tanto el Régimen de Acción Concertada como el de Acción Convenida o los sucesivos planes de reordenación, se han basado en la concertación entre empresarios mineros y empresarios eléctricos. Todos tienen en común la fijación de cuotas de producción como requisito indispensable para que las empresas mineras pudiesen acceder a las ayudas estatales, y el compromiso de compra para que las empresas eléctricas pudiesen beneficiarse de las diferentes compensaciones ofrecidas. Al igual que ocurría con las Decisiones sobre intervenciones financieras en favor del carbón, los requisitos contemplados en los diferentes Planes de reordenación también han experimentado cambios en el tiempo en un intento de mejorar la efectividad de los mismos y evitar el fraude, estableciéndose como contraprestación -a las empresas mineras- la obligación de presentar un plan de ajuste de actividad y un informe financiero de la empresa. La efectividad de los mismos, en el caso de León, se aleja considerablemente de la mayoría de los objetivos propuestos. Tan sólo se ha podido observar una reducción del empleo minero, aunque no en los porcentajes acordados en los diferentes planes. 
La puesta en marcha de los planes de reordenación pone de manifiesto, desde nuestro punto de vista, deficiencias en el diseño y ejecución de los mismos. En primer lugar, se ha perpetuado en el tiempo una distinción entre minería pública y privada que ha favorecido la discriminación de trato entre ambas. La consecuencia es que las empresas mineras públicas han proseguido en su actividad alejándose del cumplimiento de unos mínimos de eficiencia productiva; por su parte, la empresas mineras privadas se han visto privadas de unas ayudas equiparables a las de las empresas públicas que se han restringido en base a su mayor eficiencia productiva, sin tener en cuenta que las necesidades objetivas eran las mismas que para la minería pública. En segundo lugar, se han establecido cuotas de producción como único criterio para la adjudicación de las ayudas siendo más adecuado un criterio que tenga en cuenta otros parámetros como los costes de producción.

\section{BIBLIOGRAFÍA GENERAL}

AYRAL, M. (1995) "Tendencias del sector energético a nivel europeo", Economía Industrial, $\mathrm{n}^{\circ}$ 302, pp. 31-44.

Cifuentes Gonzalez, J. (1993) "La minería leonesa del carbón ante el mercado único europeo", II Curso de Economía Leonesa. Universidad de León, pp. 117-148.

Coll MARTIN, S.; C. SUdRIA I TRYAY (1987) El carbón en España (1770-1961). Una historia económica. Madrid: Turner.

CORTINA GARCIA, J. (1995) "La planificación energética de España", Economía Industrial, $\mathrm{n}^{\circ}$ 302, pp. 45-70.

CORTIzo AlVAREZ, T. (1978) "Las cuencas mineras leonesas. Aproximación a un estudio geográfico", Hornaguera, $n^{\circ} 206,207,212,209,211,213$. León.

GARCIA DELGADO, J.L. (1971) "La minería del carbón en España durante la primera Guerra Mundial. Análisis del proceso de acumulación de capital y de los principales problemas planteados", Revista de Trabajo, n 35-36.

- (1971) "Algunas conclusiones sobre la política de protección y la economía hullera asturiana en el primer tercio del siglo XX", Revista de Historia Económica, $\mathrm{n}^{\circ} 2$. Actas del I/ Congreso de Historia Económica, Facultad de Ciencias Económicas, Universidad de Alcalá de Henares (17-19 de Diciembre).

IRANZO MARTIN, J.E. (1984) "El sector energético español. Realidades y posibilidades", Papeles de Economía Española, n² 21, pp. 271-287. 
- (1992) "El sector energético español", Papeles de Economía Española, nº 50, pp. 73-180.

LuQue Cabal, J. y SierRa LoPez, R. (1995) "Tendencias del sector del carbón en la UE", Economía Industrial, n 302, pp. 123-130.

Memorias CARBUNION. Varios años.

Memorias anuales de UNESA. 1986-1999.

OTERO, J.M. y F. TRUJILlo (1983) "Estructura del consumo energético en España", Papeles de Economía Española, n 14, pp. 52-72.

Plan de futuro de la minería del carbón y desarrollo alternativo de las comarcas mineras. Madrid, 4 de febrero de 1997. Ministerio de Industria.

Plan de modernización, racionalización y reestructuración y de reducción de actividad de la industria del carbón española 1194-1997. Septiembre de 1997. Ministerio de industria.

PLAN 1998-2005. Plan 1998-2005 de la minería del carbón y Plan de Desarrollo Alternativo de las cuencas mineras. 1997. pp. 200-218. Revista Asturiana de Economía, $\mathrm{n}^{\circ} 10$. Oviedo: Universidad de Oviedo.

RAVELA MESA, T. (1991) "La estructura energética española en el periodo 1973-1988", Documentos de Trabajo, no 31. Facultad de Ciencias Económicas y Empresariales. Universidad de La Laguna.

SEN RODRIGUEZ, L.C. (1993) La minería leonesa del carbón 1764-1959. Una historia económica. León: Universidad de León.

SERVEN, L. (1989) "La empresa pública en un sector estratégico. HUNOSA", Papeles de Economía Española, n 38, pp. 383-338.

VÁzQUEZ. J.A. (1992) "La economía asturiana: en la encrucijada de los nuevos ajustes", Papeles de Economía Española, n 51, pp. 169-181.

VÁzQUeZ, J.A y S.R. MARTINEZ (1992) "El carbón en Asturias", Papeles de Economía Española, $\mathrm{n}^{\circ}$ 50, pp. 181-185.

\section{LEGISLACIÓN COMUNITARIA}

DOCE C 94/46. Resolución sobre el carbón y el mercado interior de la energía. 13.04.92.

DOCE C 180/26. Comunicación a los Estados miembros por la que se fijan las orientaciones para los programas operativos o las subvenciones globales que les invita a elaborar dentro de una iniciativa Comunitarias de transformaciones económicas de las zonas mineras del carbón (RECHAR II). 01.07.94. 
DOCE C 310/3. Pregunta escrita E-600/93 de Hiltrud Breyer a la Comisión (1 de abril de 1993) Asunto. Ayudas estatales al carbón y a la energía nuclear. 07.11.94.

DOCE C 88/88. Subvenciones estatales a favor de la industria del carbón. Proyecto de Decisión de la Comisión (COM (85) 525 Final). 14.04.96.

DOCE C 214. Dictamen del Comité Económico y Social sobre la "Comunicación de la Comisión Energía para el futuro. Fuentes de energía renovables Libro Blanco para una Estrategia y un Plan de Acción Comunitarios. 10.07.98.

DOCE C 315. Dictamen del Comité de las Regiones sobre la "Comunicación de la Comisión, Energía para el futuro. Fuentes de energía renovables. Libro Blanco para una Estrategia y un Plan de Acción Comunitarios. 13.10.98.

DOCE L 480/65. Decisión 3/65 CECA du 17.02.65. relative au régime Communautaire des interventions de États membres en faveur de l'industrie houlière. 25.02.65.

DOCE L 261/1. Prolongeant la durie de validité de la décision 3-65 du 17.02.65 relative au régime Communautaire des interventions de États membres en faveur de l'industrie houlière. 28.10.67.

DOCE L 3/7. Decision 3/71 CECA de la Commision du 22 décembre 1970 relative au régime Communautaire des interventions de États membres en faveur de l'industrie houlière. 05.01.71.

DOCE L 259/36. Decision de la Commision du 25 juillet 1973 relative aux charbons á coke et cokes destinés á la siderurgie de la Communauté. 15.09.73.

DOCE L 154. Decisión 528/76 CECA de 25 de febrero de 1976 relativa al régimen Comunitario de las intervenciones de los Estados miembros en favor de la industria hullera. 15.06.76.

DOCE $L 178 / 2$. Octroi de prêts de reconversion à taux $d$ 'intérêt réduis en application de l'article 56 du traité CECA en faveur des investissements créant de nouvelles possibilités d'emploie dans le zones touchées par un delin des activités des industries du charbon et de l'acier. 27.07.77.

DOCE L 335/8. Decisión 3501/85 CECA de la Commision du 11 dècembre 1985 modificant la Décisión $n^{\circ}$ 528/76 CECA relative au régime Communautaire des interventions des Estats membres en faveur de l'industrie houllière. 11.12.85.

DOCE L 335/8. Dècision $n^{\circ} 3501 / 85$ CECA de la Commision du 11 Décembre 1985 modifiant la décision $\mathrm{n}^{\circ} 528 / 76$ CECA relative au régime 
Communautaire des interventions des États membres en faveur de I' industrie houllière. 13.12.85.

DOCE L 177/86. Decisión 2064/86 CECA de la Comisión, de 30 de junio de 1986, relativa al régimen Comunitario de las intervenciones de los Estados miembros a favor de la industria hullera. 01.07.86.

DOCE L 241/16. Decisión de la Comisión de 31 de julio de 1987 por la que se autoriza a España para conceder ayudas en favor de la industria hullera durante 1987. 25.08.87.

DOCE L 38/39. Décision de la Commision du 24 janvier 1989 autorisant ló troi par l'espagne d'aides en faveur de l'industrie houllière en 1989. 10.02.89.

DOCE L 64/15. Decisión de la Comisión de 22 de febrero de 1989 por la que se autoriza la concesión de ayudas a al industria hullera por parte del Reino de España en el año 1986. 08.03.89.

DOCE L 105/19. Decisión de la Comisión de 20 de diciembre de 1989 relativa a una intervención financiera Complementaria de España a favor de la industria hullera en 1988 y 1987. 25.04.90.

DOCE L 329/12. Decisión 3632/93 CECA de la Comisión de 28 de diciembre de 1993 relativa al régimen Comunitario de las intervenciones de los Estados miembros en favor de la industria del carbón. 30.12.93.

DOCE L 49. Decisión $n^{\circ} 341 / 94$ CECA de la Comisión, por la que se establecen las disposiciones de aplicación de la Decisión 3632/93 CECA relativa al régimen Comunitario de las intervenciones de los Estados miembros en favor de la industria del carbón. 19.02.94.

SEC (91) 1983 Final. Informe de la Comisión al Consejo sobre la aplicación a la mitad de su vigencia de la Decisión $n^{\circ}$ 2064/86 CECA relativa al régimen Comunitario de las intervenciones de los Estados miembros en favor de la industria del carbón durante el período Comprendido entre 1987 y 1990.11 .11 .91$.

SEC (92) 2553 Final. Comunicación de la Comisión. solicitud de dictamen conforme del Consejo en consulta al Comité CECA, con arreglo al artículo 95 del Tratado CECA sobre un proyecto de Decisión de la Comisión relativa al nuevo régimen Comunitario de ayudas estatales a la industria del carbón. 27.01.93.

SEC(97) 1766 FINAL. INFORME DE LA COMISION. El mercado de los combustibles sólidos en la Comunidad en 1996 y perspectivas para 1997. 03.10.1997. 


\section{LEGISLACIÓN NACIONAL}

Decreto de 9 de agosto 1974, núm. 2485/74 (Presidencia). CARBON MINERAL. Régimen de concierto para su producción. BOE n² 218. 11.09. 1974.

Real Decreto de 16 de enero de 1981, núm. 234/81 (Presidencia). Carbón mineral. Régimen de convenios a medio plazo para su producción. BOE $\mathrm{n}^{\circ} 46$ de 23.02.81.

Orden de 23 de julio de 1987, por la que se regulan las compensaciones de OFICO por suministro, transporte y almacenamiento de carbones destinados a centrales térmicas. BOE $\mathrm{n}^{\circ} 178$ de 27.07.87

Orden de 31 de octubre de 1990 por la que se regulan las compensaciones de los costes por adquisición de carbón de origen subterráneo que excedan a los que derivan de los contemplados en los contratos a largo plazo con centrales térmicas. BOE n 267 de 07.11.90.

Orden de 14 de febrero de 1992 por la que se modifican las normas de cálculo de las compensaciones de oficio por suministro, transporte y almacenamiento de carbones destinados a centrales térmicas durante el año 1990 y siguientes. BOE n 46 de 22.02.92.

Orden de 25 de noviembre de 1993 de modificación de la Orden de 14 de febrero de 1992, por la que se establece el sistema de reducción de coste de las empresas eléctricas por la adquisición de carbón nacional que ofrezca garantía de suministro en cumplimiento de la disposición final primera del Real Decreto 1821/1991. BOE n 296 de 11.11.93.

Orden de 30 de abril de 1993 complementaria de la Orden de 31 de octubre de 1990, sobre compensaciones a empresas eléctricas explotadoras de centrales térmicas de carbón. BOE n 113 de 12.05.93.

Resolución de 20 de julio de 1994, de la Secretaría General de la Energía y Recursos Minerales, por la que se dictan normas en aplicación de los previsto en la Orden de 30 de abril de 1993, que establece una compensación para fomento del empleo en cuencas mineras del carbón. BOE $n^{\circ} 185$ de 04.08.94.

Orden de 6 de julio de 1994 por la que establece un plazo para la aplicación de las compensaciones previstas en las disposiciones que regularon el Plan de Reordenación de la Minería del Carbón para las empresas sin contrato-programa. BOE $\mathrm{n}^{\circ} 165$ de 12.07.94.

Orden de 20 de diciembre de 1994 por la que se establece el sistema de retribución del coste de las empresas eléctricas por la adquisición de carbón nacional en el marco de la aplicación al sector carbonero español de la Decisión 3632/93 CECA, de 28 de diciembre. 
Real Decreto 2203/1995, de 28 de diciembre, sobre los costes específicos derivados de las ayudas a la minería del carbón. BOE $\mathrm{n}^{\circ} 311$ de 29.12.95.

Orden de 5 de febrero de 1996 por la que se regulan las ayudas destinadas a la cobertura de los costes de explotación y se establece el Régimen Transitorio para las compensaciones anteriormente existentes. BOE $n^{\circ}$ 33 de 07.02.96.

Orden de 16 de febrero de 1996 sobre ayudas destinadas a impulsar la reactivación económica de las comarcas mineras del carbón. BOE $n^{\circ} 47$ de 23.02.96.

Orden de 29 de febrero de 1996 por la que se regulan las ayudas destinadas a la cobertura de los costes financieros de los "stocks" de carbón que se produzcan en las centrales térmicas y que excedan de los definidos como estratégicos. BOE de 07.03.93.

Orden de 1 de agosto de 1996 por la que se regulan las ayudas a la cobertura de cargas excepcionales para la minería del carbón, BOE $n^{\circ}$ 190 de 07.08.96.

Orden de 26 de febrero de 1997 por la que se modifica la de 5 de febrero de 1996 por la que se regulan las ayudas destinadas a la cobertura de los costes de explotación y se establece el Régimen Transitorio para las compensaciones anteriormente existentes. BOE $n^{\circ} 62$ de 13.03.97.

Real Decreto 2020/1997, de 26 de diciembre, por el que se establece un régimen de ayudas para la minería del carbón y el desarrollo alternativo de las zonas mineras. BOE $n^{\circ} 312$ de 30.12.97.

Orden de 18 de febrero de 1998 sobre ayudas destinadas a cubrir cargas excepcionales vinculadas a planes de modernización, reestructuración y racionalización de la actividad de las empresas mineras del carbón. BOE $n^{\circ} 48$ de 25.02.98.

Orden de 19 de octubre de 1998 por la que se regulan las ayudas destinadas a la financiación de existencias de carbón en centrales térmicas superiores a las cuantías necesarias para cubrir setecientas veinte horas de funcionamiento. BOE ${ }^{\circ} 257$ de 27.1098.

Orden de 6 de marzo de 1998 por la que se establecen las bases reguladoras para la concesión de ayudas dirigidas a proyectos empresariales generadores de empleo, que promuevan el desarrollo alternativo de las zonas mineras. BOE $\mathrm{n}^{\circ} 59$ de 10.103.98.

Real Decreto 492/1998, de 27 de marzo, por el que se aprueba el Estatuto del Instituto para la Reestructuración de la Minería del Carbón y Desarrollo Alternativo de las Cuencas Mineras. BOE $n^{\circ} 75$ de 28.03.98. 
Real Decreto 1561/1998, de 17 de julio, por el que se modifica el Real Decreto 2020/1997, de 26 de diciembre, por el que se establece un régimen de ayudas para la minería del carbón y el desarrollo alternativo de las zonas mineras. BOE $n^{\circ} 187$ de 06.08.98.

Orden de 19 de octubre de 1998 por la que se regulan las ayudas al transporte de carbón autóctono entre cuencas mineras. BOE $n^{\circ} 257$ de 27.10.98.

Real Decreto 2818/1998, de 23 de diciembre, sobre producción de energía eléctrica por instalaciones abastecidas por recursos o fuentes de energía renovables, residuos y cogeneración. BOE $n^{\circ} 312$ de 30.12.98. 